PROCEEDINGS OF THE

AMERICAN MATHEMATICAL SOCIETY

Volume 140, Number 10, October 2012, Pages 3365-3375

S 0002-9939(2012)11195-5

Article electronically published on April 24, 2012

\title{
HIGHLY-TRANSITIVE ACTIONS OF SURFACE GROUPS
}

\author{
DANIEL KITROSER
}

(Communicated by Daniel Ruberman)

\begin{abstract}
A group action is said to be highly-transitive if it is $k$-transitive for every $k \geq 1$. The main result of this thesis is the following:

Main Theorem. The fundamental group of a closed, orientable surface of genus $>1$ admits a faithful, highly-transitive action on a countably infinite set.

From a topological point of view, finding a faithful, highly-transitive action of a surface group is equivalent to finding an embedding of the surface group into $\operatorname{sym} \mathbb{Z}$ with a dense image. In this topological setting, we use methods that were originally developed for densely embedding surface groups in locally compact groups.
\end{abstract}

\section{INTRODUCTION}

A permutation group $G \leq \operatorname{Sym}(X)$ is called $k$-transitive if it is transitive on ordered $k$-tuples of distinct elements and highly-transitive if it is $k$-transitive for every $k \in \mathbb{N}$. When $G$ is given as an automorphism group, it can often be verified that the given action is highly-transitive. This is the case for any subgroup of $\operatorname{Sym}(X)$ which contains all finitely supported permutations, where $X$ is any infinite set. Other examples include groups such as $\operatorname{Homeo}\left(S^{2}\right)$ and many of its subgroups. But for most groups the question, "what is the maximal $k$ for which the group admits a faithful, $k$-transitive action?" is wide open.

It was shown by McDonough [4 that any non-abelian free group admits a highly transitive action. Another proof which is more useful from our point of view was later given by Dixon in [3], using a Baire Category type argument.

In this paper, we prove that a surface group (the fundamental group of a closed, orientable surface of genus greater than 1) admits a faithful, highly-transitive action of countably infinite degree.

1.1. Topological interpretation. Before we continue, we introduce some notation.

Notation. We denote by $S_{\infty}=\operatorname{Sym}(\mathbb{Z})$ the full symmetric group of a countable set which we identify with the integer numbers. The action of $G \leq S_{\infty}$ on $\mathbb{Z}$ is always from the right, and the image of an element $a \in \mathbb{Z}$ under $g \in G$ is denoted by $a^{g}$. If $n \in \mathbb{N}$, then $[n]$ will denote the set of all integers between $-n$ and $n$ (including $-n$ and $n)$. For $\phi \in S_{\infty}$ and $A \subset \mathbb{Z}$ we denote

$$
U(\phi, A)=\left\{\psi \in S_{\infty}|\psi|_{A}=\left.\phi\right|_{A}\right\} .
$$

Received by the editors December 15, 2010 and, in revised form, April 11, 2011.

2010 Mathematics Subject Classification. Primary 20B22, $20 \mathrm{~B} 35$.

The author was partially supported by ISF grant 888/07. 
We define a group topology on $S_{\infty}$ where a basis is given by the sets $\left\{U(\phi,[n]) \mid \phi \in S_{\infty}, n \in \mathbb{N}\right\}$. It is easy to see that if $\phi \in S_{\infty}$ and $A \subset \mathbb{Z}$ is any finite set, then $U(\phi, A)$ is open in this topology. Notice that this topology is the restriction to $S_{\infty}$ of the topology of pointwise convergence on the space of all functions $\mathbb{Z} \rightarrow \mathbb{Z}$. We now define a metric on $S_{\infty}$ : for all $\phi, \psi \in S_{\infty}$ we set $d(\phi, \psi)=0$ if $\phi=\psi$ and $d(\phi, \psi)=2^{-m}$ if $\phi \neq \psi$ and $m$ is the largest natural number such that $\left.\phi\right|_{[m]}=\left.\psi\right|_{[m]}$ and $\left.\phi^{-1}\right|_{[m]}=\left.\psi^{-1}\right|_{[m]}$. A straightforward proof shows that this metric is complete and generates the topology on $S_{\infty}$.

The problem of finding highly-transitive subgroups of $S_{\infty}$ can now be approached via the following proposition.

Proposition 1.1. Let $G \leq S_{\infty}$. Then $G$ is highly-transitive if and only if $G$ is dense in $S_{\infty}$.

Proof. Suppose $G$ is dense in $S_{\infty}$ and let $k \in \mathbb{N}$. We show that $G$ is $k$-transitive. Let $\left(a_{1}, \ldots, a_{k}\right),\left(b_{1}, \ldots, b_{n}\right)$ be two $k$-tuples of distinct elements of $\mathbb{Z}$. Since $S_{\infty}$ is $k$-transitive, there exists $\psi \in S_{\infty}$ such that $a_{i}^{\psi}=b_{i}$ for every $i=1, \ldots, k$ and since $G$ is dense in $S_{\infty}$, there exists $\phi \in G \cap U\left(\psi,\left\{a_{1}, \ldots, a_{k}\right\}\right)$. Thus, $\phi$ is an element of $G$ that takes every $a_{i}$ to $b_{i}$ as required. Conversely, we assume that $G$ is highly-transitive and show that $G$ intersects every basic neighborhood in $S_{\infty}$. Let $\psi \in S_{\infty}$ and $n \in \mathbb{N}$. Since $G$ is $(2 n+1)$-transitive, there exists $\phi \in G$ such that $i^{\phi}=i^{\psi}$ for all $i=-n, \ldots, n$ and so, $\phi \in G \cap U(\psi,[n])$.

1.2. The main result. In view of Proposition 1.1, the main result can be reformulated as follows.

Theorem 1.2 (Main Theorem). Let $\Gamma=\pi_{1}\left(\Sigma_{g}\right)$ be the fundamental group of an orientable surface of genus $\geq 2$. Then there exists a dense subgroup of $S_{\infty}$ which is isomorphic to $\Gamma$.

The methods used in this work to obtain dense embeddings of surface groups into $S_{\infty}$ are analogous to those used in 2 and 1 to show that if a locally compact group contains a dense, free subgroup of every finite rank $>1$, then it contains a dense surface group of every genus $>1 . S_{\infty}$ is not locally compact but as we shall see, by proving that $S_{\infty}$ has a dense free subgroup of every finite rank $>1$ with the additional property that certain elements of that free group generate a non-discrete cyclic group, we can apply the same methods in our setting.

\section{Dense Embeddings of Free Groups}

As a first step to proving Theorem 1.2 we prove the following.

Theorem 2.1. Let $r \geq 2$, let $w_{i}=w_{i}\left(\tau_{1}, \ldots, \tau_{r}\right)(i \in \mathbb{N})$ be cyclically reduced words in the free group on $\left\{\tau_{1}, \ldots, \tau_{n}\right\}$ and assume that $w_{i}$ is not a power of $\tau_{j}$ for any $i$ and $j$. Then, there exist $\tau_{1}, \ldots, \tau_{r} \in S_{\infty}$ such that $F=\left\langle\tau_{1}, \ldots, \tau_{r}\right\rangle$ is a dense, rank $r$ free subgroup of $S_{\infty}$ and such that $\left\langle w_{i}\left(\tau_{1}, \ldots, \tau_{r}\right)\right\rangle$ is non-discrete for every $i \in \mathbb{N}$.

We prove the theorem using Baire Category Theorem.

Definition 2.2. Let $X$ be a topological space. A subset of $X$ which is a countable intersection of dense, open sets is called residual or generic. 
Baire's theorem states that in a non-trivial, complete metric space, a residual set is dense. Since they are dense and closed under countable intersections, residual sets in a complete metric space can be thought of as being "large". Dixon showed in [3] that if we denote

$$
U=\left\{\left(\tau_{1}, \ldots, \tau_{r}\right) \in S_{\infty}^{r} \mid\left\langle\tau_{1}, \ldots, \tau_{r}\right\rangle \text { is dense in } S_{\infty}\right\}
$$

then the set of elements in $U$ that freely generate a free group is residual in $\bar{U}$. We use a somewhat different setting than Dixon in order to prove the existence of the desired dense free subgroups. Fix $\sigma \in S_{\infty}$ to be the shift permutation, i.e. $\forall a \in \mathbb{Z}: a^{\sigma}=a+1$. We show that for every $n \geq 1$, the set of elements $\left(\tau_{1}, \ldots, \tau_{n}\right) \in S_{\infty}^{n}$ such that $\left\langle\sigma, \tau_{1}, \ldots, \tau_{n}\right\rangle$ has the properties stated in Theorem 2.1 is residual in $S_{\infty}^{n}$.

Lemma 2.3. Let $\gamma \in S_{\infty}$. $\langle\gamma\rangle \leq S_{\infty}$ is non-discrete if and only if the orbits of $\langle\gamma\rangle$ are all finite and of unbounded length.

Proof. Assume first that $\langle\gamma\rangle$ has an infinite orbit $\Delta$ and let $a \in \Delta$. Then, $\langle\gamma\rangle \cap$ $U(1,\{a\})=\{1\}$ and so $\langle\gamma\rangle$ is discrete. Now assume that the lengths of the orbits of $\langle\gamma\rangle$ are uniformly bounded. Let $m$ be the product of the lengths of the orbits of $\langle\gamma\rangle$. So, $\gamma^{m}=1$; thus, $\langle\gamma\rangle$ is finite and hence discrete.

Conversely, suppose that the orbits of $\langle\gamma\rangle$ are finite and of unbounded length. To prove that $\langle\gamma\rangle$ is non-discrete it is enough to show that every basic neighborhood of the identity contains some non-trivial power of $\gamma$. Let $n \in \mathbb{N}$ and let $\Delta=\bigcup_{i=1}^{k} \Delta_{i}$ be a finite union of orbits of $\langle\gamma\rangle$ such that $[n] \subset \Delta$. By hypothesis, all the $\Delta_{i}$ 's are finite. If we set $m=\prod_{i=1}^{k}\left|\Delta_{i}\right|$, then for every $a \in \Delta$ (and in particular for every $a \in[n]$ ) we have that $a^{\gamma^{m}}=a$; and since the orbit lengths of $\langle\gamma\rangle$ are unbounded, there is an orbit of $\langle\gamma\rangle$ which is longer than $m$, so $\gamma^{m}$ is not the identity element. Thus, $\gamma^{m} \neq 1$ is an element of the pointwise stablilizer $U(1,[n])$. Since such stabilizers form a basis at the identity, we are finished.

Definition 2.4. Let $\gamma_{1}, \ldots, \gamma_{n} \in S_{\infty}$ and let $w=w\left(\gamma_{1}, \ldots, \gamma_{n}\right)$ be any word. If $w=w_{1} w_{2} \cdots w_{n}$, where $w_{i} \in\left\{\gamma_{1}^{ \pm 1}, \ldots, \gamma_{n}^{ \pm 1}\right\}$, then the trace of an element $a \in \mathbb{Z}$ under $w$ is the ordered set

$$
\operatorname{tr}_{w}(a)=\left\{a, a^{w_{1}}, a^{w_{1} w_{2}}, \ldots, a^{w_{1} w_{2} \cdots w_{n}}=a^{w}\right\}
$$

Lemma 2.5. Fix $n \in \mathbb{N}$ and let $w=w\left(\sigma, \tau_{1}, \ldots, \tau_{n}\right)$ be a reduced word which is not a conjugate of a power of $\sigma$. Then the following sets are residual:

(1) $\mathcal{F}=\left\{\left(\tau_{1}, \ldots, \tau_{n}\right) \in S_{\infty}^{n} \mid\left\langle\sigma, \tau_{1}, \ldots, \tau_{n}\right\rangle\right.$ is a free group of rank $\left.n\right\}$.

(2) $\mathcal{D}=\left\{\left(\tau_{1}, \ldots, \tau_{n}\right) \in S_{\infty}^{n} \mid\left\langle\sigma, \tau_{1}, \ldots, \tau_{n}\right\rangle\right.$ is dense $\}$.

(3) $\mathcal{N}=\left\{\left(\tau_{1}, \ldots, \tau_{n}\right) \in S_{\infty}^{n} \mid\left\langle w\left(\sigma, \tau_{1}, \ldots, \tau_{n},\right)\right\rangle\right.$ is non-discrete $\}$.

Proof. (1) Let $v$ be a reduced, non-trivial word on $n+1$ letters and consider the set

$$
\mathcal{F}_{v}=\left\{\left(\tau_{1}, \ldots, \tau_{n}\right) \in S_{\infty}^{n} \mid v\left(\sigma, \tau_{1}, \ldots, \tau_{n}\right) \neq 1\right\}
$$

If we prove that $\mathcal{F}_{v}$ is open and dense in $S_{\infty}^{n}$ for every $v$ as above, then $\mathcal{F}$ is residual since $\mathcal{F}=\bigcap_{v \neq 1} \mathcal{F}_{v}$. Obviously, $\mathcal{F}_{v}$ is open as the inverse image of the open set $S_{\infty} \backslash\{1\}$ under the continuous mapping $\left(\tau_{1}, \ldots, \tau_{n}\right) \mapsto v\left(\sigma, \tau_{1}, \ldots, \tau_{n}\right)$. To prove that $\mathcal{F}_{v}$ is dense, let $\left(\phi_{1}, \ldots, \phi_{n}\right) \in S_{\infty}^{n}$ and let $m \in \mathbb{N}$. We prove that there exists $\left(\tau_{1}, \ldots, \tau_{n}\right) \in \mathcal{F}_{v}$ such that $\left.\tau_{i}\right|_{[m]}=\left.\phi_{i}\right|_{[m]}$ for every $1 \leq i \leq n$. Write

$$
v\left(\sigma, \tau_{1}, \ldots, \tau_{n}\right)=\sigma^{r_{1}} v_{1} \sigma^{r_{2}} v_{2} \cdots \sigma^{r_{k}} v_{k} \sigma^{r_{k+1}}
$$


where $r_{i} \in \mathbb{Z}$ and $v_{i} \in\left\{\tau_{1}^{ \pm 1}, \ldots, \tau_{n}^{ \pm 1}\right\}$ for every $i$. We will define the permutations $\tau_{1}, \ldots, \tau_{n}$ in stages. First, choose $a_{1}, \ldots, a_{k+1} \in \mathbb{Z}$ such that the numbers $a_{1}, \ldots, a_{k+1}, a_{1}+r_{1}, \ldots, a_{k+1}+r_{k+1}$ are all distinct and define $\left(a_{i}+r_{i}\right)^{v_{i}}=a_{i+1}$ (e.g. if $v_{1}=\tau_{5}^{-1}$ define $\left(a_{1}+r_{1}\right)^{\tau_{5}^{-1}}=a_{2}$ or equivalently, define $\left.a_{2}^{\tau_{5}}=a_{1}+r_{1}\right)$. At this point, every $\tau_{i}$ is defined on a finite set of points. Note that since all the integers involved so far in the definition of the $\tau_{i}$ 's are distinct and $v$ is reduced, $\tau_{i}$ can be extended to a permutation of $\mathbb{Z}$, i.e. in our definition for it so far, $\tau_{i}$ does not send distinct points to the same point. We now have that $a_{1}^{v}=a_{k+1}+r_{k+1}$ and in particular, $v$ is not trivial. We choose $a_{1}, \ldots, a_{k+1}$ so that $\tau_{i}$ sends $b \in[m]$ to $b^{\phi_{i}}$. Explicitly, choose

$$
a_{1}, \ldots, a_{k+1} \in \mathbb{Z} \backslash\left([m] \cup\left(\bigcup_{i}[m]^{\phi_{i}}\right) \cup\left(\bigcup_{j}[m]-r_{j}\right) \cup\left(\bigcup_{i, j}[m]^{\phi_{i}}-r_{j}\right)\right) .
$$

Note that we can always choose $a_{1}, \ldots, a_{k+1}$ in the manner described since we are only excluding a finite set of integers that we cannot choose from. Now every $\tau_{i}$ is defined on $[m]$ and on some other elements $\left\{b_{1}, \ldots, b_{\ell}\right\} \subset\left\{a_{1}, \ldots, a_{k}, a_{1}+\right.$ $\left.r_{1}, \ldots, a_{k+1}+r_{k+1}\right\}$, sending them to $\left\{c_{1}, \ldots, c_{\ell}\right\}$ respectively. Finally, choose any bijection

$$
f_{i}: \mathbb{Z} \backslash\left([m] \cup\left\{b_{1}, \ldots, b_{\ell}\right\}\right) \rightarrow \mathbb{Z} \backslash\left([m]^{\phi_{i}} \cup\left\{c_{1}, \ldots, c_{\ell}\right\}\right)
$$

and define

$$
x^{\tau_{i}}= \begin{cases}x^{\phi_{i}}, & x \in[m], \\ c_{j}, & x=b_{j} \text { for some } j, \\ x^{f_{i}}, & \text { else. }\end{cases}
$$

So we have that every $\tau_{i}$ is a permutation which lies in the basic neighbourhood of $\phi_{i}$ defined by $[m]$ such that $v\left(\sigma, \tau_{1}, \ldots, \tau_{n}\right)$ is non-trivial.

(2) Fix some $k \in \mathbb{N}$ and for every two $k$-tuples $\mathbf{x}=\left(x_{1}, \ldots, x_{k}\right)$ and $\mathbf{y}=$ $\left(y_{1}, \ldots, y_{k}\right)$ of distinct integers consider the set

$$
\mathcal{D}_{\mathbf{x}, \mathbf{y}}=\left\{\begin{array}{l|l}
\left(\tau_{1}, \ldots, \tau_{n}\right) \in S_{\infty}^{n} & \begin{array}{l}
\exists \phi \in\left\langle\sigma, \tau_{1}, \ldots, \tau_{n}\right\rangle: \\
\forall 1 \leq j \leq k: x_{j}^{\phi}=y_{j}
\end{array}
\end{array}\right\}
$$

Notice that if $\left(\tau_{1}, \ldots, \tau_{n}\right) \in \bigcap_{\mathbf{x}, \mathbf{y}} \mathcal{D}_{\mathbf{x}, \mathbf{y}}$, where $\mathbf{x}$ and $\mathbf{y}$ range over all $k$-tuples of distinct integers, then $\left\langle\sigma, \tau_{1}, \ldots, \tau_{n}\right\rangle$ is $k$-transitive. Thus, by Proposition 1.1 we have that

$$
\mathcal{D}=\bigcap_{k \in \mathbb{N}} \bigcap_{\mathbf{x}, \mathbf{y}} \mathcal{D}_{\mathbf{x}, \mathbf{y}}
$$

and we are left to show that $\mathcal{D}_{\mathbf{x}, \mathbf{y}}$ is open and dense for every $\mathbf{x}$ and $\mathbf{y}$ as above.

First, fix $\mathbf{x}=\left(x_{1}, \ldots, x_{k}\right), \mathbf{y}=\left(y_{1}, \ldots, y_{k}\right)$ as above and let $\left(\tau_{1}, \ldots, \tau_{n}\right) \in \mathcal{D}_{\mathbf{x}, \mathbf{y}}$. By definition there exists $\phi \in\left\langle\sigma, \tau_{1}, \ldots, \tau_{n}\right\rangle$ such that $x_{i}^{\phi}=y_{i}$ for every $i=$ $1, \ldots, k$. Let us write $\phi=v\left(\sigma, \tau_{1}, \ldots, \tau_{n}\right)$, where $v=v\left(\sigma, \tau_{1}, \ldots, \tau_{n}\right)$ is a word on $\left\{\sigma^{ \pm 1}, \tau_{1}^{ \pm 1}, \ldots, \tau_{n}^{ \pm 1}\right\}$ and define $A=\bigcup_{i=1}^{k} \operatorname{tr}_{v}\left(x_{i}\right) . A$ is finite and so the set

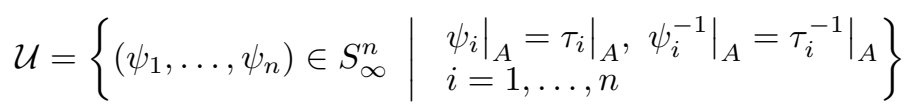

is an open neighbourhood of $\left(\tau_{1}, \ldots, \tau_{n}\right)$ contained in $\mathcal{D}_{\mathbf{x}, \mathbf{y}}$. Indeed, if $\left(\psi_{1}, \ldots, \psi_{n}\right) \in$ $\mathcal{U}$ take $\xi=v\left(\sigma, \psi_{1}, \ldots, \psi_{n}\right) \in\left\langle\sigma, \psi_{1}, \ldots, \psi_{n}\right\rangle$. Then by the definition of $\mathcal{U}$, we have 
that $\xi$ acts the same as $\phi=v\left(\sigma, \tau_{1}, \ldots, \tau_{n}\right)$ on $x_{i}$ and in particular, $\xi$ sends $x_{i}$ to $y_{i}$. This shows that $\mathcal{D}_{\mathbf{x}, \mathbf{y}}$ is open.

Now we prove that $\mathcal{D}_{\mathbf{x}, \mathbf{y}}$ is dense. Let $\left(\phi_{1}, \ldots, \phi_{n}\right) \in S_{\infty}^{n}$ and $m \in \mathbb{N}$. Let $r \in \mathbb{N}$ be such that $x_{j}+r \notin[m]$ and $y_{j}+r \notin[m]^{\phi_{1}}$ for every $1 \leq j \leq k$. Let

$$
f: \mathbb{Z} \backslash\left([m] \cup\left\{x_{1}+r, \ldots, x_{k}+r\right\}\right) \rightarrow \mathbb{Z} \backslash\left([m]^{\phi_{1}} \cup\left\{y_{1}+r, \ldots, y_{k}+r\right\}\right)
$$

be any bijection and define

$$
x^{\tau_{1}}= \begin{cases}x^{\phi_{1}}, & x \in[m], \\ y_{j}+r, & x=x_{j}+r \text { for some } 1 \leq j \leq k, . \\ x^{f}, & \text { otherwise. }\end{cases}
$$

Now defining $\tau_{i}=\phi_{i}$ for every $2 \leq i \leq n$, we get that $\left(\tau_{1}, \ldots, \tau_{n}\right)$ is an element of the basic neighbourhood of $\left(\phi_{1}, \ldots, \phi_{n}\right)$ defined by $[m]$. Also, the permutation $\xi=\sigma^{r} \tau_{1} \sigma^{-r} \in\left\langle\sigma, \tau_{1}, \ldots, \tau_{n}\right\rangle$ sends each $x_{j}$ to $y_{j}$; thus, $\left(\tau_{1}, \ldots, \tau_{n}\right) \in \mathcal{D}_{\mathbf{x}, \mathbf{y}}$.

(3) By Lemma 2.3 we can equivalently write

$$
\mathcal{N}=\left\{\left(\tau_{1}, \ldots, \tau_{n}\right) \in S_{\infty}^{n} \mid \begin{array}{l}
\text { The orbits of }\left\langle w\left(\sigma, \tau_{1}, \ldots, \tau_{n}\right)\right\rangle \text { are all finite } \\
\text { and of unbounded length. }
\end{array}\right\} .
$$

Thus, if we define for every $t \in \mathbb{N}$ and $a \in \mathbb{Z}$ :

$$
\begin{aligned}
& \mathcal{U}_{t}=\left\{\left(\tau_{1}, \ldots, \tau_{n}\right) \in S_{\infty}^{n} \mid\left\langle w\left(\sigma, \tau_{1}, \ldots, \tau_{n}\right)\right\rangle \text { has an orbit of length } \geq t\right\}, \\
& \mathcal{V}_{a}=\left\{\left(\tau_{1}, \ldots, \tau_{n}\right) \in S_{\infty}^{n} \mid \text { the orbit of } a \text { under }\left\langle w\left(\sigma, \tau_{1}, \ldots, \tau_{n}\right)\right\rangle \text { is finite }\right\},
\end{aligned}
$$

we get that $\mathcal{N}=\left(\bigcap_{t \in \mathbb{N}} \mathcal{U}_{t}\right) \cap\left(\bigcap_{a \in \mathbb{Z}} \mathcal{V}_{a}\right)$.

We now show that $\mathcal{U}_{t}$ and $\mathcal{V}_{a}$ are open and dense for every $t \in \mathbb{N}$ and $a \in$ $\mathbb{Z}$. Let $\left(\tau_{1}, \ldots, \tau_{n}\right) \in \mathcal{U}_{t}$ and let $b \in \mathbb{Z}$ be an element belonging to an orbit of $\left\langle w\left(\sigma, \tau_{1}, \ldots, \tau_{n}\right)\right\rangle$ of length $\geq t$. Thus, $b, b^{w}, b^{w^{2}}, \ldots, b^{w^{t-1}}$ are all distinct. Let $\Delta=\bigcup_{i=1}^{k-1} \operatorname{tr}_{w^{i}}(b)$. The set

$$
\left\{\begin{array}{l|l}
\left(\psi_{1}, \ldots, \psi_{n}\right) \in S_{\infty}^{n} & \begin{array}{l}
\left.\psi_{i}\right|_{\Delta}=\left.\tau_{i}\right|_{\Delta},\left.\psi_{i}^{-1}\right|_{\Delta}=\left.\tau_{i}^{-1}\right|_{\Delta} \\
i=1, \ldots, n
\end{array}
\end{array}\right\}
$$

is an open neighbourhood of $\left(\tau_{1}, \ldots, \tau_{n}\right)$ which is contained in $\mathcal{U}_{t}$; hence, $\mathcal{U}_{t}$ is open.

Now, take $\left(\tau_{1}, \ldots, \tau_{n}\right) \in \mathcal{V}_{a}$ and let $\Delta=\left\{a_{1}, \ldots, a_{s}\right\}$ be the finite orbit of $\left\langle w\left(\sigma, \tau_{1}, \ldots, \tau_{n}\right)\right\rangle$ containing $a$. Similarly

$$
\left\{\begin{array}{l|l}
\left(\psi_{1}, \ldots, \psi_{n}\right) \in S_{\infty}^{n} & \begin{array}{l}
\left.\psi_{i}\right|_{\Delta}=\left.\tau_{i}\right|_{\Delta},\left.\psi_{i}^{-1}\right|_{\Delta}=\left.\tau_{i}^{-1}\right|_{\Delta} \\
i=1, \ldots, n
\end{array}
\end{array}\right\}
$$

is an open neighbourhood of $\left(\tau_{1}, \ldots, \tau_{n}\right)$ which is contained in $\mathcal{V}_{a}$.

To prove that $\mathcal{U}_{t}$ is dense, let $\left(\phi_{1}, \ldots, \phi_{n}\right) \in S_{\infty}^{n}$ and $m \in \mathbb{Z}$. In (1) we in fact showed that we can define $\tau_{1}, \ldots, \tau_{n} \in S_{\infty}$ such that for every finite set $A \subset \mathbb{Z}$ we have that $\left.\tau_{i}\right|_{A}$ acts in any way we please and there exists some $b \in \mathbb{Z}$ such that $b^{w} \neq b$, where $w=w\left(\sigma, \tau_{1}, \ldots, \tau_{n}\right)$. By repeating the same argument we can find $\tau_{1}, \ldots, \tau_{n} \in S_{\infty}$ such that $\left.\tau_{i}\right|_{[m]}=\left.\phi_{i}\right|_{[m]}$ for every $1 \leq i \leq n$ and such that there exists $b \in \mathbb{Z}$ such that $b, b^{w}, b^{w^{2}}, \ldots, b^{w^{t-1}}$ are all distinct; i.e., $\left\langle w\left(\sigma, \tau_{1}, \ldots, \tau_{n}\right)\right\rangle$ has an orbit of length $\geq t$.

Finally, we prove that $\mathcal{V}_{a}$ is dense. Since $\left\langle w\left(\sigma, \tau_{1}, \ldots, \tau_{n}\right)\right\rangle$ has the same orbit structure as the cyclic group generated by any conjugate of $w$, we can assume without loss of generality that $w$ is a cyclically reduced word that is not a power of $\sigma$. Let $\left(\phi_{1}, \ldots, \phi_{n}\right) \in S_{\infty}^{n}$ and $m \in \mathbb{Z}$. We need to define permutations $\tau_{1}, \ldots, \tau_{n} \in S_{\infty}$ 
such that $\left(\tau_{1}, \ldots, \tau_{n}\right) \in \mathcal{V}_{a}$ and every $\tau_{i}$ agrees with $\phi_{i}$ on $[m]$. This condition can be thought of in the following way: $\tau_{i}$ is already defined on $[m]$ for every $i$ and $\tau_{i}^{-1}$ is already defined on $[m]^{\phi_{i}}$ for every $i$ (they act the same as $\phi_{i}$ and $\phi_{i}^{-1}$ respectively) and we are left to define $\tau_{i}$ on $\mathbb{Z} \backslash[m]$ (and $\tau_{i}^{-1}$ on $\mathbb{Z} \backslash[m]^{\phi_{i}}$ ) in such a way that the orbit of $a$ under $\left\langle w\left(\sigma, \tau_{1}, \ldots, \tau_{n}\right)\right\rangle$ will be finite. First we write $w\left(\sigma, \tau_{1}, \ldots, \tau_{n}\right)=w_{1} w_{2} \cdots w_{k}$. Now, we start by applying the positive and negative powers of $w$ to $a$ letter by letter:

$$
\stackrel{w_{\ell}}{\longrightarrow} c \stackrel{w_{\ell+1}}{\longrightarrow} \ldots \stackrel{w_{n-1}}{\longrightarrow} a_{-1} \stackrel{w_{n}}{\longrightarrow} a=a_{0} \stackrel{w_{1}}{\longrightarrow} a_{1} \stackrel{w_{2}}{\longrightarrow} \ldots \stackrel{w_{s-1}}{\longrightarrow} b \stackrel{w_{s}}{\longrightarrow},
$$

where $b \in \mathbb{Z}$ is the first element such that we need to apply to it the permutation $w_{s}$ and $w_{s}$ is not yet defined on $b$; that is, $w_{s}=\tau_{i}$ for some $i$ and $b \notin[m]$ or $w_{s}=\tau_{i}^{-1}$ for some $i$ and $b \notin[m]^{\phi_{i}}$. Note that if such an element $b$ does not exist, then, since by hypothesis at least one of the letters $w_{1}, \ldots, w_{k}$ is not $\sigma$, the orbit of $a$ under $\left\langle w\left(\sigma, \phi_{1}, \ldots, \phi_{n}\right)\right\rangle$ is contained in $[m] \cup\left(\bigcup_{i=1}^{n}[m]^{\phi_{i}}\right)$, hence is finite, and we can just take $\tau_{i}=\phi_{i}$ for every $i$. We can thus assume that such a $b$ exists. Similarly, $c \in \mathbb{Z}$ is the first element we reach when applying letter by letter the negative powers of $w$ to $a$ such that we need to apply to $c$ the permutation $w_{\ell}^{-1}$, and $w_{\ell}^{-1}$ is not yet defined on $c$. As before, we can assume without loss of generality that such an element $c$ exists. By hypothesis, $w$ is cyclically reduced and so the word $w_{s} w_{s+1} \cdots w_{k} w_{1} \cdots w_{\ell}$ is reduced. Also, by their definition, $w_{s}, w_{\ell} \neq \sigma^{ \pm 1}$. We wish to define $\tau_{1}, \ldots, \tau_{n}$ in such a way that $b^{w_{s} w_{s+1} \cdots w_{k} w_{1} \cdots w_{\ell}}=c$ (and of course fulfilling the condition that $\tau_{i}$ agrees with $\phi_{i}$ on $[m]$ ). By repeating the argument made in $(1)$, we can find two distinct elements $d_{1}, d_{2} \in \mathbb{Z}$ that do not lie in $[m]$ or any $[m]^{\phi_{i}}$ such that $w_{s+1} \cdots w_{k} w_{1} \cdots w_{\ell-1}$ sends $d_{1}$ to $d_{2}$ and define $b^{w_{s}}=d_{1}, d_{2}^{w_{\ell}}=c$. From this we get that $b^{w_{s} \cdots w_{k} w_{1} \cdots w_{l}}=d_{1}^{w_{s+1} \cdots w_{k} w_{1} \cdots w_{\ell}}=d_{2}^{w_{\ell}}=c$; thus the orbit of $a$ is finite. Now, every $\tau_{i}$ is defined on $[m]$ and maybe on finitely many more elements. Again, exactly as we did in (1), we can extend the definition of $\tau_{i}$ to $\mathbb{Z}$ and get permutations $\tau_{1}, \ldots, \tau_{n}$ satisfying the required conditions.

Proof of Theorem 2.1. Let $j \in\{1, \ldots, r\}$ and set $\tau_{j}=\sigma$. Then by Lemma 2.5, the set

$W_{i}=\left\{\begin{array}{l|l}\left(\tau_{1}, \ldots, \tau_{j-1}, \tau_{j+1}, \ldots, \tau_{r}\right) \in S_{\infty}^{r-1} & \begin{array}{l}\left\langle\tau_{1}, \ldots, \tau_{r}\right\rangle \text { is a dense, rank } r \text { free } \\ \text { subgroup and }\left\langle w_{i}\left(\tau_{1}, \ldots, \tau_{r}\right)\right\rangle \\ \text { is non-discrete }\end{array}\end{array}\right\}$

is residual and so $\bigcap_{i \in \mathbb{N}} W_{i}$ is residual and, in particular, not empty.

\section{Eventually faithful Sequences}

Definition 3.1. Let $G$ and $H$ be groups. A sequence $\left\{f_{n}\right\}_{n=1}^{\infty}$ of homomorphisms from $G$ to $H$ is eventually faithful if for every $g \in G$ there exists $n_{0} \in \mathbb{N}$ such that $g \notin \operatorname{ker}\left(f_{n}\right)$ for all $n \geq n_{0}$.

In order to prove the main theorem, we will need to produce eventually faithful sequences of homomorphisms from surface groups to free groups. The following constructions appear in 2 and 1 . Let $\Gamma=\Gamma_{2 r}$ be the surface group of genus $2 r$ $(r \geq 1)$. We have the following presentation for $\Gamma$ :

$$
\Gamma=\left\langle a_{1}, a_{1}^{\prime}, \ldots, a_{r}, a_{r}^{\prime}, b_{1}, b_{1}^{\prime}, \ldots, b_{r}, b_{r}^{\prime} \mid\left[a_{1}, a_{1}^{\prime}\right] \cdots\left[a_{r}, a_{r}^{\prime}\right]\left[b_{r}^{\prime}, b_{r}\right] \cdots\left[b_{1}^{\prime}, b_{1}\right]\right\rangle .
$$


Let $x=\left[a_{1}, a_{1}^{\prime}\right] \cdots\left[a_{r}, a_{r}^{\prime}\right]$ and let $h: \Gamma \rightarrow \Gamma$ be the Dehn twist around $x$, i.e.

$$
\begin{array}{lll}
h\left(a_{i}\right)=a_{i}, & & h\left(b_{i}\right)=x b_{i} x^{-1}, \\
h\left(a_{i}^{\prime}\right)=a_{i}^{\prime}, & h\left(b_{i}^{\prime}\right)=x b_{i}^{\prime} x^{-1} .
\end{array}
$$

Let $F$ be the free group on $2 r$ free generators $\left\{\phi_{1}, \phi_{1}^{\prime}, \ldots, \phi_{r}, \phi_{r}^{\prime}\right\}$ and let $k: \Gamma \rightarrow F$ be the homomorphism defined by

$$
\begin{aligned}
& k\left(a_{i}\right)=k\left(b_{i}\right)=\phi_{i}, \\
& k\left(a_{i}^{\prime}\right)=k\left(b_{i}^{\prime}\right)=\phi_{i}^{\prime} .
\end{aligned}
$$

Consider the map that folds the genus $2 r$ surface that has $\Gamma$ as its fundamental group across the curve corresponding to $x$ (this curve separates the surface into two equal parts). The image of this map is a surface of genus $r$ with one boundary component, so it has $F$ as its fundamental group. $k$ is the homomorphism induced on the fundamental groups by this folding map (see Figure 1). Denote $f_{n}=k \circ h^{n}$.

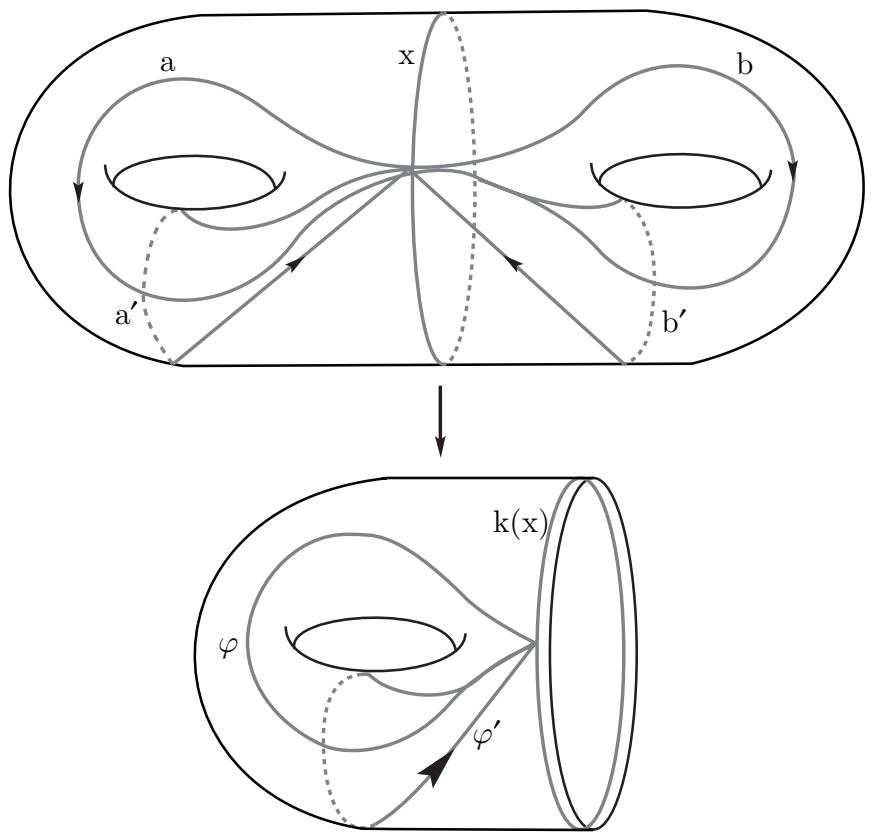

Figure 1

Lemma 3.2 (Breuillard, Gelander, Souto, Storm [2]). The sequence $\left\{f_{n}\right\}_{n=1}^{\infty}$ is eventually faithful.

We now construct an eventually faithful sequence of homomorphisms from an odd genus surface group into a free group. Let $\Gamma=\Gamma_{2 r+1}$ be a surface group of genus $2 r+1(r \geq 1)$ with the presentation

$\Gamma=\left\langle a_{1}, a_{1}^{\prime}, \ldots, a_{r}, a_{r}^{\prime}, b, b^{\prime}, c_{1}, c_{1}^{\prime}, \ldots, c_{r}, c_{r}^{\prime} \mid\left[a_{1}, a_{1}^{\prime}\right] \cdots\left[a_{r}, a_{r}^{\prime}\right]\left[b^{\prime}, b\right]\left[c_{1}^{\prime}, c_{1}\right] \cdots\left[c_{r}^{\prime}, c_{r}\right]\right\rangle$. 
Denote $x=\left[a_{1}, a_{1}^{\prime}\right] \cdots\left[a_{r}, a_{r}^{\prime}\right] b^{\prime}$ and let $F$ be the free group on $2 r+1$ free generators $\left\{\phi_{1}, \phi_{1}^{\prime}, \ldots, \phi_{r}, \phi_{r}^{\prime}, \tau\right\}$. Let $\delta: \Gamma \rightarrow \Gamma$ and $\zeta: \Gamma \rightarrow \Gamma$ be Dehn twists around $x$ and $b^{\prime}$ respectively, that is,

$$
\begin{aligned}
\delta\left(a_{i}\right) & =a_{i} & \zeta\left(a_{i}\right) & =a_{i} \\
\delta\left(a_{i}^{\prime}\right) & =a_{i}^{\prime} & \zeta\left(a_{i}^{\prime}\right) & =a_{i}^{\prime} \\
\delta(b) & =x b & \zeta(b) & =b\left(b^{\prime}\right)^{-1} \\
\delta\left(b^{\prime}\right) & =b^{\prime} & \zeta\left(b^{\prime}\right) & =b^{\prime} \\
\delta\left(c_{i}\right) & =x c_{i} x^{-1} & \zeta\left(c_{i}\right) & =c_{i} \\
\delta\left(c_{i}^{\prime}\right) & =x c_{i}^{\prime} x^{-1} & \zeta\left(c_{i}^{\prime}\right) & =c_{i}^{\prime} .
\end{aligned}
$$

Notice that $\delta$ and $\zeta$ commute.

Let $k: \Gamma \rightarrow F$ be the map induced by folding the $2 r+1$ surface across the curves $x$ and $b^{\prime}$ (these curves separate the surface into two surfaces of genus $r$ and two boundary components). Explicitly,

$$
\begin{aligned}
& k\left(a_{i}\right)=\phi_{i} \\
& k\left(a_{i}^{\prime}\right)=\phi_{i}^{\prime} \\
& k(b)=1 \\
& k\left(b_{i}^{\prime}\right)=\tau \\
& k\left(c_{i}\right)=\phi_{i} \\
& k\left(c_{i}^{\prime}\right)=\phi_{i}^{\prime}
\end{aligned}
$$

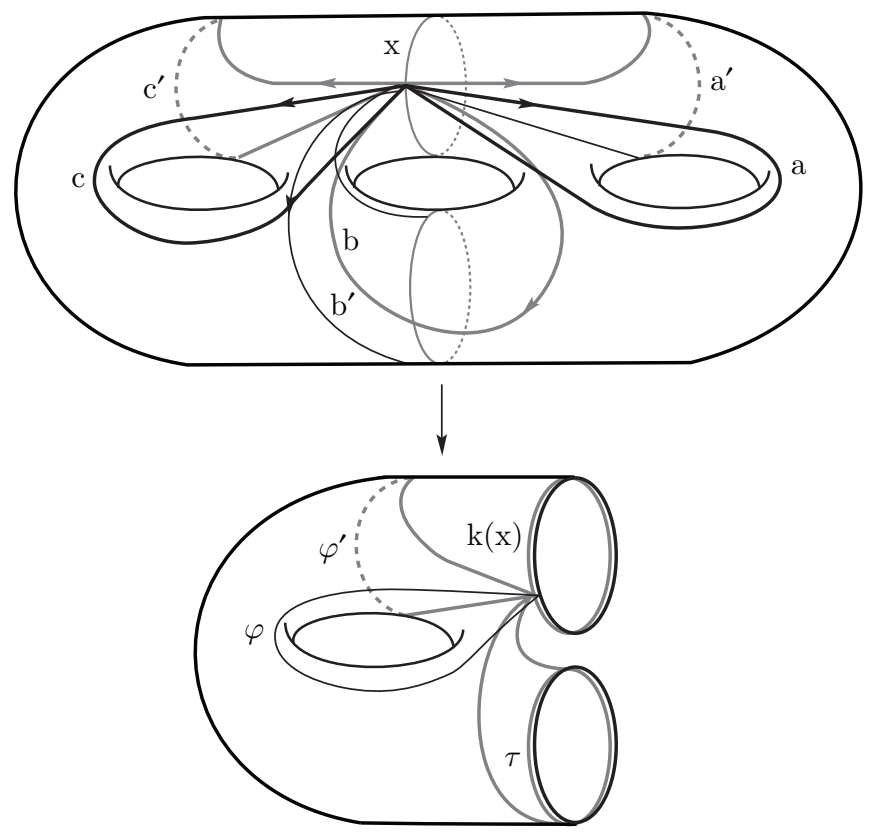

Figure 2 
(see Figure 2). Finally, denote $\rho_{n}=k \circ(\delta \circ \zeta)^{n}$.

Lemma 3.3 (Barlev, Gelander [1). The sequence $\left\{\rho_{n}\right\}_{n=1}^{\infty}$ is eventually faithful.

\section{Proof of the Main theorem}

We will need the following results.

Lemma 4.1. Let $\phi, \psi \in S_{\infty}$ be such that both $\langle\phi\rangle$ and $\langle\psi\rangle$ are non-discrete. Then $\langle(\phi, \psi)\rangle \leq S_{\infty}^{2}$ is non-discrete.

Proof. We prove that every basic neighbourhood of $(1,1)$ contains a non-trivial element of $\langle(\phi, \psi)\rangle$. Let $m \in \mathbb{N}$. Let $\Delta=\bigcup_{i=1}^{\ell} \Delta_{i}$ and $\Gamma=\bigcup_{i=1}^{k} \Gamma_{i}$ be finite unions of orbits of $\langle\phi\rangle$ and $\langle\psi\rangle$ respectively such that $[m] \subset \Delta$ and $[m] \subset \Gamma$. From Proposition 2.3 we have that all the orbits of $\langle\phi\rangle$ and $\langle\psi\rangle$ are finite, and so we can define the number

$$
n=\prod_{i=1}^{\ell}\left|\Delta_{i}\right| \cdot \prod_{i=1}^{k}\left|\Gamma_{i}\right| .
$$

Notice that every element of $\Delta$ is fixed by $\phi^{n}$ and every element of $\Gamma$ is fixed by $\psi^{n}$ and in particular every $i \in[m]$ is fixed by $\phi^{n}$ and $\psi^{n}$. From Proposition 2.3 we also have that the lengths of the orbits of $\langle\phi\rangle$ and $\langle\psi\rangle$ are unbounded and in particular $\langle\phi\rangle$ and $\langle\psi\rangle$ both have an orbit of length greater than $n$, and so $\phi^{n}$ and $\psi^{n}$ are non-trivial. Thus $(\phi, \psi)^{n}$ is a non-trivial element contained in the basic neighbourhood of $(1,1)$ defined by $[m] \times[m]$.

Lemma 4.2. Let $G$ be a Hausdorff topological group and let $\gamma \in G$ such that $\langle\gamma\rangle$ is non-discrete. Then, for every $n_{0} \in \mathbb{N}$ the set $\left\{\gamma^{n} \mid n \geq n_{0}\right\}$ is dense in $\overline{\langle\gamma\rangle}$.

Proof. First we notice that since $\langle\gamma\rangle$ is non-discrete, then also $\overline{\langle\gamma\rangle}$ is non-discrete. Let $U \subset \overline{\langle\gamma\rangle}$ be open. Then we have $\gamma^{m} \in U$ for some $m \in \mathbb{Z}$. If $m \geq n_{0}$ we are done, so assume $m<n_{0}$. Now, if we denote

$$
\begin{aligned}
U^{\prime} & =U \gamma^{-m}, \\
U^{\prime \prime} & =U^{\prime} \cap\left(U^{\prime}\right)^{-1},
\end{aligned}
$$

then $U^{\prime \prime}$ is an open symmetric identity neighborhood and since $\overline{\langle\gamma\rangle}$ is non-discrete, $U^{\prime \prime}$ is not finite. Now take

$$
\widetilde{U}=U^{\prime \prime} \backslash\left\{\gamma^{k}:|k|<n_{0}-m\right\} .
$$

We have that $\widetilde{U}$ is open (since $G$ is Hausdorff) and non-empty; thus there exists $n \in \mathbb{Z}$ such that $\gamma^{n} \in \widetilde{U}$. By the definition of $\widetilde{U}$ we have that $|n| \geq n_{0}-m$. Also, since $\widetilde{U} \subset U^{\prime \prime}$ and $U^{\prime \prime}$ is symmetric it follows that $\gamma^{n}, \gamma^{-n} \in U^{\prime \prime} \subset U^{\prime}$. Hence, $\gamma^{n+m}, \gamma^{-n+m} \in U$, and since either $n+m \geq n_{0}$ or $-n+m \geq n_{0}$ we are done.

4.1. Proof of Theorem $\mathbf{1 . 2}$ for even genus. By Theorem 2.1 there exists a subgroup $F \leq S_{\infty}$ such that $F$ is dense, free with $2 r$ free generators $\phi_{1}, \phi_{1}^{\prime}, \ldots, \phi_{r}, \phi_{r}^{\prime} \in$ $S_{\infty}$ and such that $\left\langle\left[\phi_{1}, \phi_{1}^{\prime}\right] \cdots\left[\phi_{r}, \phi_{r}^{\prime}\right]\right\rangle$ is non-discrete. Denote $\gamma=\left[\phi_{1}, \phi_{1}^{\prime}\right] \cdots\left[\phi_{r}, \phi_{r}^{\prime}\right]$ and $\Omega=\overline{\langle\gamma\rangle}$. Let $\Gamma=\Gamma_{2 r}$ be a surface group of genus $2 r(r \geq 1)$ with the presentation

$$
\Gamma=\left\langle a_{1}, a_{1}^{\prime}, \ldots, a_{r}, a_{r}^{\prime}, b_{1}, b_{1}^{\prime}, \ldots, b_{r}, b_{r}^{\prime} \mid\left[a_{1}, a_{1}^{\prime}\right] \cdots\left[a_{r}, a_{r}^{\prime}\right]\left[b_{r}^{\prime}, b_{r}\right] \cdots\left[b_{1}^{\prime}, b_{1}\right]\right\rangle .
$$


Define for every $\omega \in \Omega$ a homomorphism $f_{\omega}: \Gamma \rightarrow S_{\infty}$ by

$$
\begin{aligned}
f_{\omega}\left(a_{i}\right) & =\phi_{i}, \\
f_{\omega}\left(a_{i}^{\prime}\right) & =\phi_{i}^{\prime}, \\
f_{\omega}\left(b_{i}\right) & =\omega \phi_{i} \omega^{-1}, \\
f_{\omega}\left(b_{i}^{\prime}\right) & =\omega \phi_{i}^{\prime} \omega^{-1} .
\end{aligned}
$$

Since every $\omega \in \Omega$ commutes with $\gamma$, this defines a homomorphism. Indeed, for every $\omega \in \Omega$ :

$$
\begin{aligned}
& f_{\omega}\left(\left[a_{1}, a_{1}^{\prime}\right] \cdots\left[a_{r}, a_{r}^{\prime}\right]\left[b_{r}^{\prime}, b_{r}\right] \cdots\left[b_{1}^{\prime}, b_{1}\right]\right) \\
& =\underbrace{\left[\phi_{1}, \phi_{1}^{\prime}\right] \cdots\left[\phi_{r}, \phi_{r}^{\prime}\right]}_{\gamma} \omega \underbrace{\left[\phi_{r}^{\prime}, \phi_{r}\right] \cdots\left[\phi_{1}^{\prime}, \phi_{1}\right]}_{\gamma^{-1}} \omega^{-1} \\
& =\gamma \omega \gamma^{-1} \omega^{-1}=1 .
\end{aligned}
$$

Every element of $\Omega$ corresponds to a homomorphism $\Gamma \rightarrow S_{\infty}$ whose image contains $F$; hence the image is dense. We are left to show that at least one of those homomorphisms is also faithful. The space $\Omega$ is a completely metrizable space, so it suffices to show that $\chi=\left\{\omega \in \Omega \mid f_{\omega}\right.$ is faithful $\}$ is residual in $\Omega$.

For every $g \in \Gamma \backslash\{1\}$ denote $\chi_{g}=\left\{\omega \in \Omega \mid f_{\omega}(g) \neq 1\right\}$. Notice that

$$
\chi=\bigcap_{g \in \Gamma \backslash\{1\}} \chi_{g} .
$$

From Lemma 3.2 the sequence $\left\{f_{\gamma^{n}}\right\}_{n \in \mathbb{N}}$ is eventually faithful, and so for every $g \in \Gamma \backslash\{1\}$ there exists $n_{0} \in \mathbb{N}$ such that $\gamma^{n} \in \chi_{g}$ for all $n \geq n_{0}$. By Lemma4.2 we have that $\left\{\gamma^{n} \mid n \geq n_{0}\right\}$ is dense in $\Omega$, and so $\chi_{g}$ is dense in $\Omega$. $\chi_{g}$ is also open as the inverse image of the open set $S_{\infty} \backslash\{1\}$ under the continuous map $\omega \rightarrow f_{\omega}(g)$. This shows that $\chi$ is residual in $\Omega$.

4.2. Proof of Theorem $\mathbf{1 . 2}$ for odd genus. Let $\Gamma=\Gamma_{2 r+1}$ be a surface group of genus $2 r+1(r \geq 1)$ with the presentation

$$
\Gamma=\left\langle a_{1}, a_{1}^{\prime}, \ldots, a_{r}, a_{r}^{\prime}, b, b^{\prime}, c_{1}, c_{1}^{\prime}, \ldots, c_{r}, c_{r}^{\prime} \mid\left[a_{1}, a_{1}^{\prime}\right] \cdots\left[a_{r}, a_{r}^{\prime}\right]\left[b^{\prime}, b\right]\left[c_{1}^{\prime}, c_{1}\right] \cdots\left[c_{r}^{\prime}, c_{r}\right]\right\rangle
$$

Let $F \leq S_{\infty}$ be a dense, free subgroup on $2 r+1$ free generators $\phi_{1}, \phi_{1}^{\prime}, \ldots, \phi_{r}, \phi_{r}^{\prime}, \tau \in$ $S_{\infty}$ such that $\langle\tau\rangle$ and $\left\langle\left[\phi_{1}, \phi_{1}^{\prime}\right] \cdots\left[\phi_{r}, \phi_{r}^{\prime}\right] \tau\right\rangle$ are non-discrete (the existence of such a free subgroup is assured by Theorem 2.1). Denote $\gamma=\left[\phi_{1}, \phi_{1}^{\prime}\right] \cdots\left[\phi_{r}, \phi_{r}^{\prime}\right] \tau$ and $\Omega=\overline{\langle(\gamma, \tau)\rangle} \subset S_{\infty}^{2}$. For every $(\psi, \xi) \in \Omega$ we define a homomorphism $f_{(\psi, \xi)}: \Gamma \rightarrow$ $S_{\infty}$ by setting

$$
\begin{aligned}
& f_{(\psi, \xi)}\left(a_{i}\right)=\phi_{i}, \quad f_{(\psi, \xi)}(b)=\psi \xi^{-1}, \quad f_{(\psi, \xi)}\left(c_{i}\right)=\psi \phi_{i} \psi^{-1}, \\
& f_{(\psi, \xi)}\left(a_{i}^{\prime}\right)=\phi_{i}^{\prime}, \quad f_{(\psi, \xi)}\left(b^{\prime}\right)=\tau, \quad f_{(\psi, \xi)}\left(c_{i}^{\prime}\right)=\psi \phi_{i}^{\prime} \psi^{-1} \text {. }
\end{aligned}
$$


Since every $(\psi, \xi) \in \Omega$ commutes with $(\gamma, \tau)$ we have that $f_{(\psi, \xi)}$ is well defined as a homomorphism because

$$
\begin{aligned}
& f_{(\psi, \xi)}\left(\left[a_{1}, a_{1}^{\prime}\right] \cdots\left[a_{r}, a_{r}^{\prime}\right]\left[b^{\prime}, b\right]\left[c_{1}^{\prime}, c_{1}\right] \cdots\left[c_{r}^{\prime}, c_{r}\right]\right) \\
& =\left[\phi_{1}, \phi_{1}^{\prime}\right] \cdots\left[\phi_{r}, \phi_{r}^{\prime}\right]\left[\tau, \psi \xi^{-1}\right] \psi \underbrace{\left[\phi_{1}^{\prime}, \phi_{1}\right] \cdots\left[\phi_{r}^{\prime}, \phi_{r}\right]}_{=\tau \gamma^{-1}} \psi^{-1} \\
& =\gamma \psi \xi^{-1} \tau^{-1} \xi \psi^{-1} \psi \tau \gamma^{-1} \psi^{-1}=\gamma \psi \xi^{-1} \tau^{-1} \xi \tau \gamma^{-1} \psi^{-1} \\
& =\gamma \psi \xi^{-1} \xi \tau^{-1} \tau \gamma^{-1} \psi^{-1}=\gamma \psi \gamma^{-1} \psi^{-1}=1 .
\end{aligned}
$$

Notice that $f_{\left(\gamma^{n}, \tau^{n}\right)}=\rho_{n}$ (as defined is Section 3) for every $n \in \mathbb{N}$, and so by Lemma 3.3. the sequence $f_{\left(\gamma^{n}, \tau^{n}\right)}$ is eventually faithful.

The image of every homomorphism $f_{\left(\gamma^{n}, \tau^{n}\right)}$ contains $F$; hence the image is dense. Finally, we show that $\chi=\left\{(\psi, \xi) \in \Omega \mid f_{(\psi, \xi)}\right.$ is faithful $\}$ is residual in the completely metrizable space $\Omega$ and, in particular, $\chi \neq \varnothing$. For every $g \in \Gamma \backslash\{1\}$ we denote $\chi_{g}=\left\{(\psi, \xi) \in \Omega \mid f_{(\psi, \xi)}(g) \neq 1\right\}$. As in the previous section, $\chi_{g}$ is open as the inverse image of an open set under a continuous map. Since $f_{\left(\gamma^{n}, \tau^{n}\right)}$ is eventually faithful there exists $n_{0} \in \mathbb{N}$ such that $f_{\left(\gamma^{n}, \tau^{n}\right)} \in \chi_{g}$ for all $n \geq n_{0}$. Since $\langle\tau\rangle$ and $\langle\gamma\rangle$ are non-discrete we get from Lemma 4.1 that $\Omega$ is non-discrete, and so by Lemma 4.2 we have that $\left\{\left(\gamma^{n}, \tau^{n}\right) \mid n \geq n_{0}\right\}$ is dense in $\Omega$. This shows that $\chi_{g}$ is dense; hence

$$
\chi=\bigcap_{g \in \Gamma \backslash\{1\}} \chi_{g}
$$

is residual.

\section{ACKNOWLEDGEMENT}

This work is the author's M.Sc. thesis. He wishes to thank his advisor, Professor Yair Glasner, for his advice, patience and guidance.

\section{REFERENCES}

1. J. Barlev and T. Gelander, Compactifications and algebraic completions of limit groups, Journal d'Analyse Mathématique 112 (2010), no. 1, 261-287. MR2763002

2. E. Breuillard, T. Gelander, J. Souto and P. Storm, Dense embeddings of surface groups, Geometry and Topology 10 (2006), 1373-1389. MR2255501 (2008b:22007)

3. J. D. Dixon, Most finitely generated permutation groups are free, Bull. London Math. Soc. 22 (1990), no. 3, 222-226. MR.1041134 (91c:20005)

4. T. P. McDonough, A permutation representation of a free group, Quart. J. Math. Oxford Ser. 28 (1977), no. 3, 353-356. MR0453869 (56:12122)

Department of Mathematics, Ben-Gurion University of The Negev, Be'er Sheva, ISRAEL

E-mail address: kitrosar@bgu.ac.il 